\title{
Heuristic algorithms for surveyor standby location planning with multiple plans
}

\author{
Rawee Suwandechochai, Wasin Padungwech* \\ Received: December 2, 2020. Revised: December 30, 2020. Accepted: December 30, 2020
}

\begin{abstract}
This paper concerns a location problem which arises from an auto insurance company which aims to meet customers' satisfaction by sending their surveyors to accident locations as promptly as possible. The goal of the problem is to find a suitable set of locations at which the surveyors stand by and to determine coverage area for each standby location by using heuristic algorithms. One challenge of this problem is that the number and locations of accidents can vary from time to time and may not be evenly distributed over a given time horizon. In this paper, a computational study is conducted to make a comparison between standby location planning strategies that involve one and two plans. For the strategies with two plans, several strategies for deciding when to switch between the plans are investigated. Experimental studies suggest that using multiple standby location plan throughout the time horizon can improve the efficiency of surveyors in terms of their total distance. Moreover, compared with usual rules of splitting dates such as weekdays versus weekends, the total distance can be reduced by switching between the location plans according to classification of dates by k-means clustering based on historical data of accident frequencies on each day.
\end{abstract}

Keywords - Systems Theory, Signal Processing, classification, insurance company, k-means clustering, location problem, p-median, surveyor.

\section{INTRODUCTION}

A location problem is an optimization problem for finding locations of facilities and warehouses. This problem has been applied to many industries including the service industries. One common location problem is the p-median problem, in which the aim is to determine a given number ( $p$ ) of locations of facilities for satisfying given demands. It was first introduced by Hakimi [1], where the problem was tackled in order to find the locations of telephone switching centers. In fact, there are many other applications in which real-life problems can be solved as the p-median problem, for example, school districts [2], bank locations [3], ambulances relocation

This research is supported by the Centre of Excellence in Mathematics, Post Graduate Education and Research Development Office of the Ministry of Higher Education, Science, Research, and Innovation, Thailand.

Rawee suwandechochai is with Department of Mathematics, Faculty of Science, Mahidol University, Bangkok, Thailand and Centre of Excellence in Mathematics, Post Graduate Education and Research Development Office of the Ministry of Higher Education, Science, Research, and Innovation, Thailand (email: rawee.suw@mahidol.ac.th).

Wasin Padungwech is with Department of Mathematics, Faculty of Science, Mahidol University, Bangkok, Thailand and Centre of Excellence in Mathematics, Post Graduate Education and Research Development Office of the Ministry of Higher Education, Science, Research, and Innovation, Thailand (*corresponding author email: wasin.pad@mahidol.ac.th).
[4], hospitals [5], blood facility locations [6].

In this paper, we consider a real-life application in an auto insurance company. The auto insurance business is highly competitive market. The company may be able to improve the services by providing quick responses to its customers. Once the accident occurs the company must send a surveyor to the accident site as quick as possible. To meet the customers' satisfaction, the auto insurance company must make decisions on how many standby locations for the surveyors they should have, where they should be situated, and also how to allocate each accident point to each standby location so that the surveyor can reach the accident point within the limited time. Unlike the location problem in many industries, another challenge for the auto insurance company is that the demand (accident point) is unknown when the standby location plan is determined which make the problem more difficult. After looking at the historical data of accidents in some real-life data, we also found that the number of accidents at each area is not evenly distributed from day to day.

The aim of this paper is to improve the auto insurance services via the strategic management of surveyor standby locations. To reach this goal, the main objective is to find the standby locations for surveyor, the number of standby locations so that the total cost is minimized. In addition, the coverage area for each standby location is also determined.

This paper allows the possibility of using different standby location plans on different days. So, in addition to finding standby locations, another challenge in this problem is to decide when to switch between standby location plans. In other words, we seek to determine several standby location plans ( 2 in this paper) and assign one of such plans to each day in a given time horizon in such a way that minimizes the total cost. This problem can be formulated as follows.

Let $G=(V, E)$ be a complete undirected graph, where $V$ represents a set of locations ("districts"). Let $c_{i j}$ be the cost (or distance) for servicing a location $j$ from a standby location $i \in V$. Let $D=\left\{1,2, \ldots, d_{\max }\right\}$ be a set of days, and $n_{j}^{d}$ the number of accident cases at location $j$ on day $d \in D$. Let $x_{i j}^{t}$ be a binary variable such that $x_{i j}^{t}=1$ if location $i$ is assigned as a standby location to location $j$ in the day cluster $t \in\{0,1\}$ (considering in this paper a solution that contains 2 standby location plans, one plan for each cluster), otherwise $x_{i j}^{t}=0$.

Let $y_{i}^{t}$ be a decision variable such that $y_{i}^{t}=1$ if location $i$ is 
used as a standby location in the day cluster $t$, and $y_{i}^{t}=0$ otherwise. Let $l_{d}$ be a decision variable that denotes the day cluster in which day $d \in D$ is contained. Let $p$ be the number of standby locations. The objective of the problem is to minimize

$$
\sum_{t=0}^{1} \sum_{i \in V} \sum_{j \in V} n_{j}^{t} c_{i j} x_{i j}^{t}
$$

subject to

$$
\begin{aligned}
& x_{i j}^{t} \leq y_{i}^{t}, \quad \forall i \in V, \forall j \in V \forall t \in\{0,1\} \\
& \sum_{i} y_{i}^{t}=p \quad \forall t \in\{0,1\} \\
& n_{j}^{0}=\sum_{d=1}^{d_{\max }} n_{j}^{d}\left(1-l_{d}\right) \\
& n_{j}^{1}=\sum_{d=1}^{d_{\max }} n_{j}^{d} l_{d} \\
& \sum_{i \in V} x_{i j}^{t} \leq n_{j}^{t}, \quad \forall j \in V, \forall t \in\{0,1\} \\
& \sum_{i \in V} x_{i j}^{t} \leq 1, \quad \forall j \in V, \forall t \in\{0,1\} \\
& M \sum_{i \in V} x_{i j}^{t} \geq n_{j}^{t}, \quad \forall j \in V, \forall t \in\{0,1\} \\
& x_{i j}^{t}, y_{i}^{t}, l_{d} \in\{0,1\}, \forall i \in V, \forall j \in V, \forall t \in\{0,1\}
\end{aligned}
$$

The objective function above is the total cost due to servicing locations with accident cases from assigned standby locations over the given time horizon. Constraint (1) ensures that location $i$ is identified as a standby location when it is assigned for servicing some location $j$. Constraint (2) specifies the number of standby locations. Constraints (3) and (4) compute the total number of accident cases at location $j$ in each day cluster. Constraints (5), (6), and (7) ensure that each location $j$ is assigned to exactly one standby location in the day cluster $t$ if it has at least one accident case in that day cluster. In constraint (7), $M$ is a sufficiently large number. Constraint (8) specifies possible values of the decision variables. Notice that this is an extension to the p-median problem as the $\mathrm{p}$-median problem can be retrieved by reducing the number of day clusters to one.

The location problem can be formulated as integer programming and mixed-integer programming depending on the constraints. It has been well known that it is an NP-hard problem. Although the exact method can be used to find the optimal solution, it is not practical when the problem is of large scale. Due to this drawback, heuristics and metaheuristic algorithms have been introduced. For example, Resende and Werneck [7] considered a multistart hybrid heuristic technique called GRASP. Other heuristic methods such as simulated annealing (SA), genetic algorithm, (GA), Particle swarm optimization (PSO), Variable Neighbourhood Search (VNS), and so on, have also been studied. Please see [8-11] for details. These works are concerned with finding the facility or depots locations according to the objectives.
Another stream of works is on the location problem for services such as ambulance and emergency medical services (EMS). Brotcorne et.al. [12] have summarized the works which are relevant to the location and relocation models for ambulance services. The similarity is in the quick response time required to provide services to customers. However, the objectives of the problem are slightly different. For example, Toregas et al. [13] developed a mathematical model for set covering problem in order to minimize the number of ambulances needed. Another objective is to maximize the coverage problem when the number of ambulances is fixed as proposed by Church and ReVelle [14]. Recently, the trend of the research is to consider the double standard model (DSM) introduced by Gendreau, Laporte, and Semet [15]. This model has been considered to solve problems in many countries as may be seen in [16]-[18].

Although our problem is a location problem for service, the objective of our problem is slightly different from those of ambulance and EMS problems. In addition, the standby locations in our problem can be changed from time to time. Thus, in practice we propose to develop more than one standby location plan and find the criteria to switch from one to another.

\section{Methods}

In this section, several methods of planning standby locations are described. Methods for planning standby locations that will be investigated in this paper can be divided into two main components: determining when a standby location plan should be switched and determining a set of standby locations.

\section{A. Strategies of switching standby location plans}

As stated earlier, using a single location plan throughout a given time horizon may not be effective. Thus, we attempt to develop a strategy that involves more than one location plan so that it is possible to switch plans at appropriately specified time. In what follows, Strategy 1 refers to a basic strategy that involves only one location plan which is used every day. This will be compared with other strategies that involve more than one location plan in order to investigate whether there is any benefit of switching plans during the time horizon.

The second strategy is to classify the daily accident data into two groups such as "busy" and "non-busy" days, and a promising location plan is then developed for each group. To implement this method, a plan is switched from one to another based on classification of the data. After investigating the pattern of our collected data, as detailed in the Computational Study section, it is obvious that the numbers of accidents during working days (Monday to Friday) and during weekends (Saturday and Sunday) are significantly different. Thus, we decide to separate the data into two groups based on this observation; this strategy will be referred to as Strategy 2a. Another possible classification of the data is to consider whether or not a given day is a holiday; a strategy developed based on this classification is called Strategy $2 b$.

Instead of using the same number of plans for all locations, we shall also investigate a strategy by which one location plan 
is used throughout the time horizon for some locations, while the remaining locations are covered by two location plans which can be switched at specified moments in time. This strategy will be referred to Strategy 2c.

The next strategy, called Strategy 2d, involves two location plans as previous strategies do, although the days are classified into two groups based on the number of accidents in various districts. More precisely, each day is associated with a data point which is an $n$-tuple of non-negative numbers, whose $i^{\text {th }}$ number is the number of accident cases in the $i^{\text {th }}$ district on the corresponding day. Such data points are then clustered by the k-means clustering algorithm [19]-[20]. Another strategy, called Strategy 2e, is similar to Strategy $2 \mathrm{~d}$ but each data point is associated to the number of accident cases in each month rather than each day.

Another strategy is to search for promising classification of the daily data points by means of a genetic algorithm (GA), in place of k-means clustering in previous strategies. For the genetic algorithm considered in this study, a chromosome (i.e. a solution) is expressed as a sequence of $n$ days, where $n$ is the number of clusters to be specified and the days are represented by integer indices $0,1,2, \ldots, 364$. For each chromosome, the corresponding clustering can be obtained by viewing the days in the chromosome as centres of data point clusters, and each data point is put into a cluster whose centre is "closest" to that data point. Here, the distance or "dissimilarity" between data points of days $d_{1}$ and $d_{2}$ is defined as the sum of squares of differences between the number of cases on those days at each location, that is,

$$
\operatorname{dist}\left(d_{1}, d_{2}\right)=\sum_{j \in V}\left(n_{j}^{d_{1}}-n_{j}^{d_{2}}\right)^{2}
$$

where $n_{j}^{d}$ is the number of accident cases at each location $j$ on day $d$. In this work, each chromosome contains 2 elements as we focus on clustering days into 2 groups. Given days $d_{l}$ and $d_{2}$ to be cluster centres, the corresponding clustering can be determined by checking whether the data point of each day is "more similar" to the data point of $d_{1}$ or $d_{2}$. More precisely, the data points are splits into 2 sets $D_{1}$ and $D_{2}$, where $D_{1}$ is the set of days $d$ such that $\operatorname{dist}\left(d_{1}, d\right) \leq \operatorname{dist}\left(d_{2}, d\right)$, i.e. it is "more similar" to day $d_{1}$ than day $d_{2}$, and $D_{2}$ contains the remaining days.

The fitness function for evaluating a chromosome in this GA is defined as follows. Let

$$
\begin{aligned}
& S_{1}=\sum_{d \in D_{1}} \operatorname{dist}\left(d_{1}, d\right) \\
& S_{2}=\sum_{d \in D_{2}} \operatorname{dist}\left(d_{1}, d\right) .
\end{aligned}
$$

The fitness function of $\left(d_{1}, d_{2}\right)$ is defined as

$$
f\left(d_{1}, d_{2}\right)=\frac{\operatorname{dist}\left(d_{1}, d_{2}\right)}{S_{1}+S_{2}} .
$$

In the GA considered in this work, uniform crossover [21] is used. The GA also involves mutation and elitism. Of each new generation, some portions are taken from the population in the previous generation. The values of relevant parameters in the experiment will be given in Section III.

\section{B. Determination of standby locations}

After clustering the data points, a set of standby locations for each cluster is constructed by one of the following ways.

1) Averaging coordinates

Given the number of standby locations, accident cases in each data cluster are divided into the specified number of groups by the k-means clustering method based on their coordinates. A standby location is then determined by computing a centre of each group, that is, averaging coordinates of locations of the accident cases in that group. Thus, in this way, each accident case is serviced by a surveyor who stands by at the centre of the corresponding group.

\section{2) P-median problem}

In this way, a set of standby locations is also constructed by the k-means clustering method as in 1). However, a further step is implemented to determine an association between standby locations and accident cases. Given the number of standby locations, accident cases are assigned to standby locations (that is, each accident case being serviced by a surveyor who stands by at the assigned location) in such a way that the total distance is minimized. This is similar to the $p$ median problem with the assumption that the set of medians have already been chosen. More formally, let $c_{i j}$ be the cost between locations (districts) $i$ and $j, f_{j}$ be the total number of cases at location $j$, and $x_{i j}$ be a decision variable such that $x_{i j}=$ 1 if an accident case at location $j$ is serviced by a surveyor who stands by at location $i$, and $x_{i j}=0$ otherwise. Let $m$ be the number of possible standby locations, and $n$ be the number of locations that have accident cases. This problem can be formulated as follows.

$$
\begin{array}{ll}
\text { Minimize } & \sum_{i=1}^{m} \sum_{j=1}^{n} f_{j} c_{i j} x_{i j} \\
\text { subject to } & \sum_{i=1}^{m} x_{i j}=1, \quad \forall j \in\{1,2, \ldots, n\}, \\
& x_{i j} \in\{0,1\}, \quad \forall i \in\{1,2, \ldots, m\} \text { and } j \in\{1,2, \ldots, n\}
\end{array}
$$

The equation in the above formulation means that each location must be serviced by one standby location.

\section{Iterative search heuristic for improving standby location plans}

After standby location plans are obtained from the methods described in Sections B.1) and B.2), it may be possible to further improve such plans by an iterative heuristic search. This section describes such a heuristic that relies on repeatedly performing data clustering of individual days and solving the p-median problem alternately.

This method proceeds as follows. Given the data points that have been clustered (for example, a strategy in Section A. may be used to obtain initial clusters), a set of standby locations for each cluster is determined by solving the p-median problem. An example is illustrated in Fig. 1, where dots represent locations and adjacent numbers represent the number of accident cases at each location on each day. In this example, 
days 1 and 3 are grouped into one cluster, and days 2 and 4 are grouped into the other cluster. For each cluster, the numbers of accident cases at each location are combined, and a set of standby locations is obtained by solving the p-median based on such total numbers.

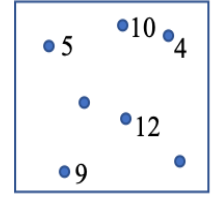

Day 1
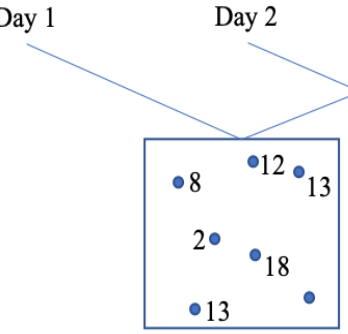

Days 1 and 3

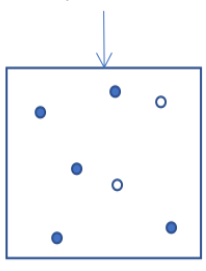

Standby location plan 1
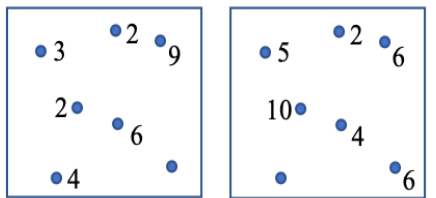

Day 3

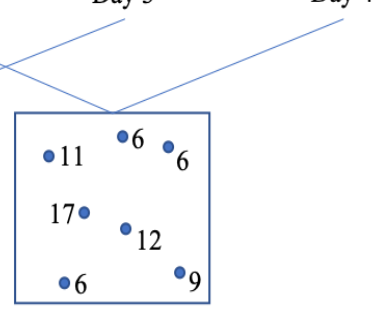

Days 2 and 4

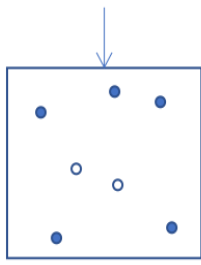

Standby location

plan 2
Fig. 1 An example of clustering data of individual days and obtaining standby location plans. Each dot represents a location, and an adjacent number represents the number of accident cases at that location on each day. White dots represent standby locations.

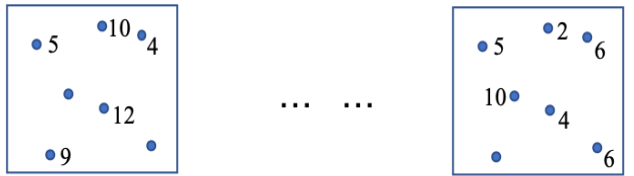

Day 1

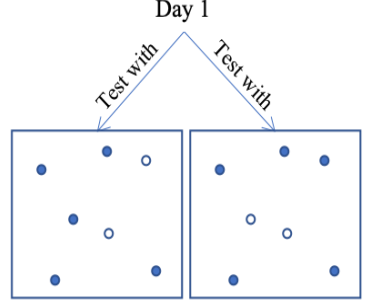

Standby location Standby location plan 1

plan 2

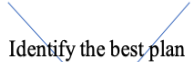

Identify the best plan

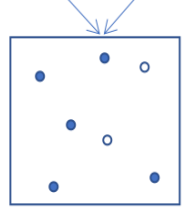

Standby location

plan 1

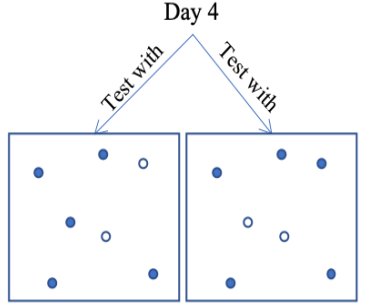

Standby location Standby location plan $1 \quad$ plan 2
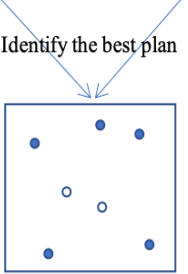

Standby location plan 2

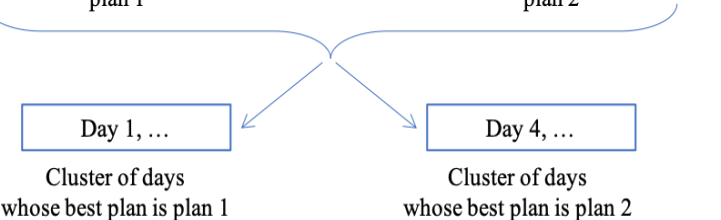

Fig. 2 A conceptual overview of re-clustering in the iterative heuristic search. Dots and numbers are as described in Fig. 1.

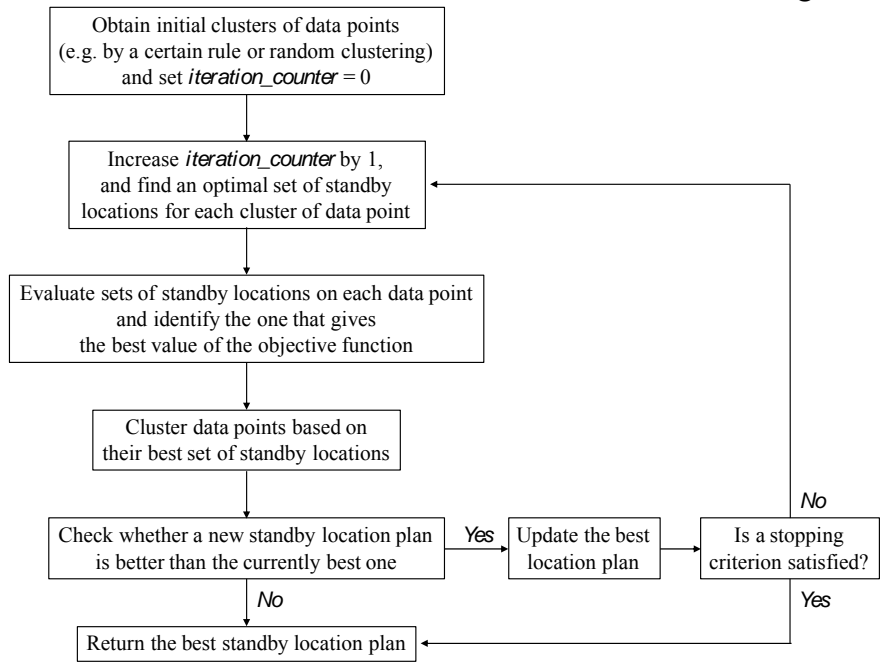

Fig. 3 A flowchart of the iterative heuristic search for improving standby location plans.

After that, each set of standby locations is evaluated with each data point, as illustrated by Fig. 2. The data points are then re-clustered based on the best standby location plan. After the new clusters are obtained, a set of standby locations for each cluster is determined again by solving the p-median problem. This process alternates between clustering given data points and determining sets of standby locations by solving the $\mathrm{p}$-median problem and continues until a stopping criterion is 
satisfied. An overview of this method is illustrated by a flowchart in Fig. 3.

\section{RESULTS AND DISCUSSION}

In our computational study, real-life data from various provinces in Thailand, provided by a prominent Thai auto insurance company, is considered. The data was collected from 113,021 accident cases during the year 2018. The data consists of the date and time period at which each accident occurred and the location (district) of the accidents, from which the number of accident cases at each location can be deduced. A set of possible standby locations (districts) in this problem contains 928 locations.

To reach our goal in this paper, the seven strategies described in Section II are compared by implementing them to the above data, and the total distance, the locations of the standby points, and how to allocate the coverage of each standby points are investigated. For the genetic algorithm (strategy 2f), the population size is 200, the mutation probability is 0.1 , the ratio of elite population is 0.01 , the crossover probability is 0.5 , the portion of population to be taken to a successive generation is 0.3 , and the maximum number of iterations is 100 .

For a fair comparison, all strategies are tested with the same number of standby locations, which is set to 119 (the number used in the existing data) in this experiment. For Strategy 2c, two location plans are implemented on a group of $20 \%$ of districts with the greatest number of accident cases throughout the year and this group is given $80 \%$ of the standby locations.

\section{A. Results of standby location plans from average coordinates}

Table 1 shows experimental results from the various options of surveyor location planning in terms of the total distance travelled by surveyors throughout the year from standby locations to assigned accident cases, where the accident cases are assigned to the standby locations based on the groups of accident locations from the k-means clustering method, according to B1) in Section III.

The results are computed from 10 experimental runs. Details of these results are shown in Table 2.

We note that the k-means clustering method can give different results in different runs as the initial cluster centroids vary from run to run. The results suggest that different ways of clustering dates into 2 groups can lead to noticeable difference in the total distance travelled by surveyors from their standby locations to assigned accident locations.

Table 1. Comparison of various strategies of standby location planning from 10 experimental runs, where standby locations are based on average coordinates in each cluster of accident locations.

\begin{tabular}{|c|c|c|c|}
\hline Strategy & Average $(\mathrm{km})$ & $\begin{array}{c}\text { Coefficient of } \\
\text { variation }\end{array}$ & $\begin{array}{c}\% \text { deviation } \\
\text { from Strategy } \\
1 *\end{array}$ \\
\hline Strategy 1 & $986,325.2$ & $2.1 \%$ & - \\
\hline
\end{tabular}

\begin{tabular}{|c|c|c|c|}
\hline $\begin{array}{c}\text { (using 1 plan) } \\
\text { (using 2 plans: } \\
\text { weekdays/weekends) }\end{array}$ & $980,176.8$ & $1.4 \%$ & $(0.62 \%)$ \\
\hline $\begin{array}{c}\text { Strategy 2b } \\
\text { (using 2 plans: non- } \\
\text { holidays/holidays) }\end{array}$ & $980,486.3$ & $2.3 \%$ & $(0.59 \%)$ \\
\hline $\begin{array}{c}\text { Strategy 2c } \\
\text { (using 2 plans for } \\
\text { locations with great } \\
\text { occurrence of } \\
\text { accidents, and 1 plan } \\
\text { for the others) }\end{array}$ & $1,460,354.0$ & $0.6 \%$ & $48.06 \%$ \\
\hline $\begin{array}{c}\text { Strategy 2d } \\
\text { (using 2 plans: daily } \\
\text { data grouped by k- } \\
\text { means) }\end{array}$ & $978,610.0$ & $1.3 \%$ & $(0.78 \%)$ \\
\hline $\begin{array}{c}\text { Strategy 2e } \\
\text { (using 2 plans: } \\
\text { monthly data } \\
\text { grouped by k-means) }\end{array}$ & $978,707.8$ & $1.3 \%$ & $(0.77 \%)$ \\
\hline $\begin{array}{c}\text { Clustering daily data } \\
\text { grouped by a genetic } \\
\text { algorithm (GA) }\end{array}$ & $978,856.5$ & $1.7 \%$ & $(0.76 \%)$ \\
\hline
\end{tabular}

*Numbers in brackets mean the results are better than Strategy 1.

More precisely, Strategies 2a, 2b, 2d, 2e, and $2 \mathrm{f}$ are superior to Strategy 1 since they result in lower total distances, whereas Strategy 2c leads to a noticeably worse result. This suggests that implementing more than one location plan can improve the effectiveness of standby locations, but it has to be coupled with an appropriate timing of switching plans.

Table 2. Total distances given by different strategies of standby location plans over 10 experimental runs, where standby locations are based on the groups of accident locations from the k-means clustering method.

\begin{tabular}{|c|c|c|c|c|c|}
\hline \multirow{2}{*}{ Strategy } & \multicolumn{5}{|c|}{ Total distance $(\mathrm{km})$} \\
\cline { 2 - 6 } & Run 1 & Run 2 & Run 3 & Run 4 & Run 5 \\
\hline 1 & $988,955.5$ & $988,346.0$ & $1,039,273.8$ & $967,563.3$ & $981,342.9$ \\
\hline 2a & $977,716.5$ & $964,145.7$ & $986,814.8$ & $988,817.8$ & $995,592.9$ \\
\hline 2b & $979,008.1$ & $992,519.0$ & $993,537.7$ & $983,040.4$ & $1,003,009.4$ \\
\hline 2c & $1,462,215.0$ & $1,462,209.9$ & $1,438,249.8$ & $1,467,353.9$ & $1,467,924.2$ \\
\hline 2d & $973,424.3$ & $982,875.5$ & $956,852.0$ & $957,637.5$ & $997,063.8$ \\
\hline 2e & $966,951.8$ & $1,003,853.6$ & $967,957.3$ & $978,530.3$ & $970,671.4$ \\
\hline GA & $976,631.8$ & $973,934.5$ & $982,127.0$ & $953,703.6$ & $1,001,140.6$ \\
\hline
\end{tabular}

Table 2. (continued).

\begin{tabular}{|c|c|c|c|c|c|}
\hline \multirow{2}{*}{ Strategy } & \multicolumn{5}{|c|}{ Total distance $(\mathrm{km})$} \\
\cline { 2 - 6 } & Run 6 & Run 7 & Run 8 & Run 9 & Run 10 \\
\hline 1 & $995,082.4$ & $978,580.2$ & $956,195.4$ & $983,885.7$ & $984,027.1$ \\
\hline
\end{tabular}


INTERNATIONAL JOURNAL OF CIRCUITS, SYSTEMS AND SIGNAL PROCESSING

\begin{tabular}{|c|r|c|c|c|l|}
\hline 2a & $989,930.6$ & $983,589.4$ & $956,877.0$ & $996,928.2$ & $961,355.5$ \\
\hline 2b & $931,859.7$ & $1,015,225.3$ & $982,362.2$ & $971,964.7$ & $952,336.5$ \\
\hline 2c & $1,466,282.7$ & $1,457,618.7$ & $1,464,968.3$ & $1,450,738.0$ & $1,465,979.9$ \\
\hline 2d & $987,609.1$ & $990,302.6$ & $982,788.7$ & $983,523.1$ & $974,023.6$ \\
\hline 2e & $996,083.9$ & $971,663.3$ & $988,352.1$ & $971,623.3$ & $971,390.8$ \\
\hline GA & $967,988.2$ & $989,154.1$ & $1,009,488.6$ & $956,338.9$ & $978,057.8$ \\
\hline
\end{tabular}

\section{B. Results of standby location plans from solving the p-} median problem

Table 3 shows experimental results from standby location planning in which accident cases are assigned to standby locations by solving the p-median problem (see Section III.B.2). The results are also computed from 10 experimental runs, the details of which are shown in Table 4.

The results in Table 3 also appears to suggest that using multiple standby location plans throughout the year can reduce the total distance travelled by surveyors compared to using only a single plan. However, when comparing the results between Table 1 and Table 3, it can be seen that the assignment between accident locations and standby locations from the p-median model can lead to lower total distance compared with the method of averaging coordinates.

Table 3. Comparison of various strategies of standby location planning from 10 experimental runs, where standby locations are determined from the $\mathrm{p}$-median problem.

\begin{tabular}{|c|c|c|c|}
\hline Strategy & $\begin{array}{c}\text { Average } \\
(\mathrm{km})\end{array}$ & $\begin{array}{c}\text { Coefficient } \\
\text { of variation }\end{array}$ & $\begin{array}{c}\% \text { deviation } \\
\text { from } \\
\text { Strategy 1 } \\
\text { with } \\
\text { averaging } \\
\text { coordinates* }\end{array}$ \\
\hline $\begin{array}{c}\text { Strategy 1 } \\
\text { (using 1 plan) }\end{array}$ & $976,574.1$ & $2.0 \%$ & $(0.99 \%)$ \\
\hline $\begin{array}{c}\text { Strategy 2a } \\
\text { (using 2 plans: } \\
\text { weekdays/weekends) }\end{array}$ & $970,145.9$ & $1.4 \%$ & $(1.64 \%)$ \\
\hline $\begin{array}{c}\text { Strategy 2b } \\
\text { (using 2 plans: non- } \\
\text { holidays/holidays) }\end{array}$ & $971,357.4$ & $2.2 \%$ & $(1.52 \%)$ \\
\hline $\begin{array}{c}\text { Strategy 2c (using 2 } \\
\text { plans for locations with } \\
\text { great occurrence of } \\
\text { accidents, and 1 plan } \\
\text { for the others) }\end{array}$ & $1,453,206.1$ & $0.7 \%$ & $47.34 \%$ \\
\hline $\begin{array}{c}\text { Strategy 2d } \\
\text { (using 2 plans: daily } \\
\text { data grouped by k- }\end{array}$ & $985,475.8$ & $2.0 \%$ & \\
\hline
\end{tabular}

\begin{tabular}{|c|c|c|c|}
\hline means) & & & \\
\hline $\begin{array}{c}\text { Strategy 2e } \\
\text { (using 2 plans: } \\
\text { monthly data } \\
\text { grouped by k- } \\
\text { means) }\end{array}$ & $974,364.7$ & $1.7 \%$ & $(1.21 \%)$ \\
\hline $\begin{array}{c}\text { Clustering daily } \\
\text { data grouped by a } \\
\text { genetic algorithm } \\
\text { (GA) }\end{array}$ & $971,324.1$ & $1.7 \%$ & $(1.52 \%)$ \\
\hline
\end{tabular}

*Numbers in brackets mean the results are better than Strategy 1 with averaging coordinates.

Table 4. Total distances given by different strategies of standby location plans over 10 experimental runs, where standby locations are determined from the p-median problem.

\begin{tabular}{|c|c|c|c|c|c|}
\hline \multirow{2}{*}{ Strategy } & \multicolumn{5}{|c|}{ Total distance (km) } \\
\cline { 2 - 7 } & Run 1 & Run 2 & Run 3 & Run 4 & Run 5 \\
\hline 1 & $981,277.1$ & $976,618.4$ & $1026,167.7$ & $955,990.6$ & $970,973.9$ \\
\hline 2a & $968,555.2$ & $952,546.3$ & $972,085.3$ & $980,769.3$ & $983,216.8$ \\
\hline 2b & $971,693.9$ & $985,062.1$ & $984,476.7$ & $973,305.7$ & $991,151.7$ \\
\hline 2c & $1,458,074.8$ & $1,453,084.7$ & $1,426,205.4$ & $1,463,760.4$ & $1,459,618.8$ \\
\hline 2d & $965,608.5$ & $1,012,440.7$ & $998,864.9$ & $944,103.6$ & $984,511.3$ \\
\hline 2e & $958,732.9$ & $1,011,578.8$ & $969,703.7$ & $965,835.3$ & $962,745.4$ \\
\hline GA & $965,454.6$ & $966,463.2$ & $973,538.4$ & $946,230.5$ & $996,467.8$ \\
\hline
\end{tabular}

Table 4. (continued).

\begin{tabular}{|c|c|c|c|c|c|}
\hline \multirow{2}{*}{ Strategy } & \multicolumn{5}{|c|}{ Total distance (km) } \\
\cline { 2 - 7 } & Run 6 & Run 7 & Run 8 & Run 9 & Run 10 \\
\hline 1 & $985,130.1$ & $963,384.7$ & 950,461 & $976,869.7$ & $978,868.1$ \\
\hline 2a & $984,693.3$ & $973,119.6$ & $947,168.8$ & $985,558.5$ & $953,746.2$ \\
\hline 2b & $924,297.8$ & $1,005,017.6$ & $967,648.9$ & $963,440.5$ & $947,479.2$ \\
\hline 2c & $1,460,770.2$ & $1,447,336.0$ & $1,454,319.9$ & $1,446,158.2$ & $1,462,732.6$ \\
\hline 2d & $996,414.2$ & $982,314.6$ & $984,139.9$ & $975,981.9$ & $1,010,378.6$ \\
\hline 2e & $998,939.3$ & $960,611.6$ & $977,973.3$ & $971,133.8$ & $966,392.9$ \\
\hline GA & $962,744.8$ & $982,657.9$ & $995,474.9$ & $949,937.1$ & $974,272.1$ \\
\hline
\end{tabular}

\section{Results of standby location plans from the iterative search heuristic}

We now attempt to further improve standby location plans by the iterative search heuristic. In this subsection, we now focus on Strategies 2a, 2b, 2d, 2e, and 2f, all of which involve 2 standby location plans over the given time horizon. Here, standby locations are no longer from averaging coordinates of locations in initial clusters, but are taken from the set of all locations given in the problem. Fig. 4 displays the results from this heuristic after a single iteration and the results after up to 10 iterations, within which the heuristic is terminated at the 
iteration in which no improvement is found. The same experiment is repeated once more on another data set (from another year 2019 provided by the same company), the results from which are shown in Fig. 5. The results show that the iterative heuristic search has the capability to improve the standby location plans across different initial clustering of individual days. Furthermore, this illustrates the fact that even though standby location plans are obtained from an exact solution of the p-median problem, they can still be improved because such an exact solution is based on initial clustering of data of individual days and an initial guess of such a clustering may not be the best.

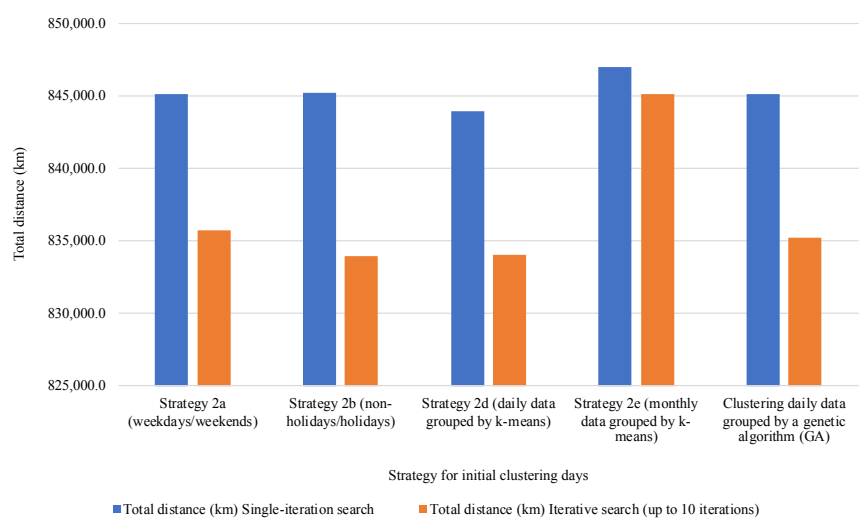

Fig. 4 Comparison of various strategies of standby location planning, where standby locations are determined from the iterative heuristic search, based on the year 2018 data set.

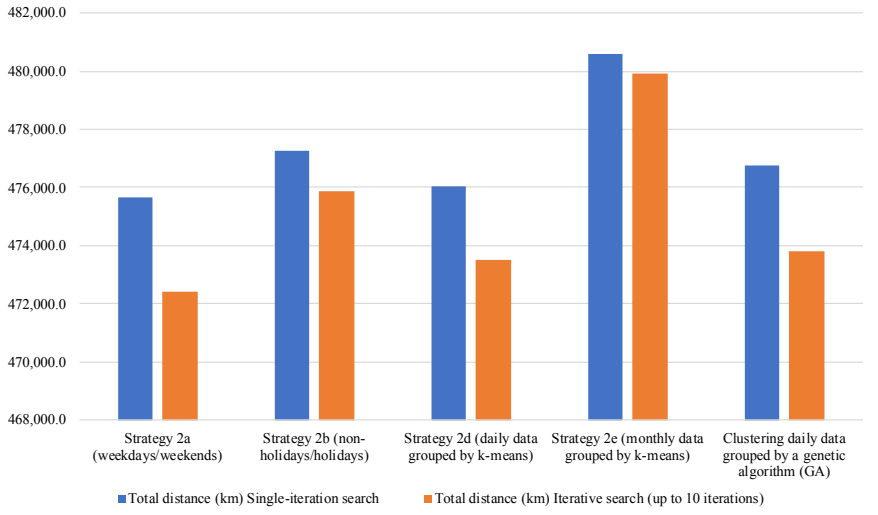

Fig. 5 Comparison of various strategies of standby location planning, where standby locations are determined from the iterative heuristic search, based on the year 2019 data set.

\section{CONCLUSIONS}

This paper concerns a location problem that arises from an auto insurance company. In particular, the problem is to determine a set of standby locations from which surveyors of the company travel to accident cases. The work in this paper was motivated from the fact that the number of accident cases may not always remain the same from day to day, and thus it may be beneficial to seek a strategy that involves more than one location plan and to switch between plans at appropriate times. In this paper, several strategies of standby location plans have been investigated and compared. Moreover, this paper suggests an iterative heuristic search technique which is based on alternately clustering data of individual days and determining an optimal set of standby locations by solving a relevant $\mathrm{p}$-median problem. Computational results show that it is possible to improve the effectiveness of surveyors by implementing more than one standby location plan throughout a time horizon being considered, although this should be accompanied by an appropriate timing of switching plans. In addition, the iterative heuristic search technique has been shown to have the capability to further improve the standby location plans and at the same time determine an appropriate standby location plan for each day.

Possible future research could involve applications of the technique to more complexed allocation problems with additional limitations desired by the service providers or the customers or could involve experimenting with other classification techniques such as in [22]. It has been illustrated by the work described here that the heuristic search technique can be a versatile procedure for applications that strives to improve an issue by iteratively upgrading the arrangement depending on a specified heuristic capability or a cost function or measure.

\section{ACKNOWLEDGMENT}

This research is supported by the Centre of Excellence in Mathematics, Post Graduate Education and Research Development Office of the Ministry of Higher Education, Science, Research, and Innovation, Thailand.

\section{REFERENCES}

[1] S. L. Hakimi, "Optimum location of switching centers and the absolute centers and medians of a graph," Oper Res, vol. 12, pp. 450-459, 1964.

[2] R. Honey, G. Rushton, P. Lononis, B. Dalziel, M. Arm-strong, S. De and P. Densham, "Stages in the adoption of a spatial decision support system for reorganizing service delivery regions," Environ Plann C, Vol. 9(1), 51-63, 1991.

[3] D. Willer, "A spatial decision support system for bank location: A case study," NCGIA Technical Report, vol. 90, no. 9, 1990.

[4] W. E. McAleer and I.A. Naqvi, "The relocation of ambulance stations: A successful case study," Eur J Oper Res, vol. 75, pp. 582-588, 1994.

[5] A. Mehrez, Z. Sinuany-Stern, A.-G. Tal, and B. Shemuel. "On the implementation of quantitative facility location models: The Case of a hospital in a rural region," J Oper Res Soc, vol. 47, pp. 612-625, 1996.

[6] D. A. Jacobs, M.N. Silan, and B.A. Clemson, "An Analysis of alternative locations and service areas of American Red Cross blood facilities," Interfaces, vol. 26, pp. 40-50, 1996.

[7] M. G. C. Resende, R. F. Werneck, "A hybrid heuristic for the p-median problem," J Heuristics, vol. 10, pp. 59-88, 2004.

[8] A. T. Murray, R. L. Church, "Applying simulated annealing to locationplanning models." J Heuristics, vol. 2, pp. 31-53, 1996.

[9] P. Hansen, N. Mladenović, D. Perez-Britos, "Variable neighborhood decomposition search," J Heuristics, vol. 7, pp. 335-350, 2001.

[10] Y.-C. Chiou, L. W. Lan, "Genetic clustering algorithms," Eur J Oper Res, vol. 135, pp. 413-427, 2001.

[11] B. Bozkaya, J. Zhang, E. Erkut, "An efficient genetic algorithm for the p-median problem," in Facility Location: Applications and Theory, Z. Drezner, H. W. Hamacher, Eds. Berlin: Springer, 2002, pp. 179-205.

[12] L. Brotcorne, G. Laporte, F. Semet, "Ambulance location and relocation models," Eur J Oper Res, vol. 147, pp. 451-463, 2003.

[13] C. Toregas, R. Swain, C. ReVelle, L. Bergman, "The location of emergency service facilities," Oper Res, vol. 19, pp. 1363-1373, 1971.

[14] R. Church, C. ReVelle, "The maximal covering location problem," Pap Reg Sci Assoc, vol. 32, pp. 101-118, 1974.

[15] M. Gendreau, G. Laporte, F. Semet, "Solving an ambulance location model by tabu search," Location Science, vol. 5, pp. 75-88, 1997. 
[16] K. F. Doerner, W. J. Gutjahr, R. F. Hartl, M. Karall, M. Reimann, "Heuristic solution of an extended double-coverage ambulance location Problem for Austria," Cent Eur J Oper Res, vol. 13, pp. 325-340, 2005.

[17] K. Schneeberger, K. F. Doerner, A. Kurz, M. Schilde, "Ambulance location and relocation models in a crisis," Cent Eur J Oper Res, vol. 24, pp. 1-27, 2016.

[18] J. C. Dibene, Y. Maldonado, C. Vera, M. de Oliveira, L. Trujillo, O. Schütze, "Optimizing the location of ambulances in Tijuana, Mexico," Comput Biol Med, vol. 80, pp. 107-115, 2017.

[19] C. Elkan, "Using the triangle inequality to accelerate kmeans," Proceedings of the 20th international conference on Machine Learning (ICML-03), pp. 147-153, 2003.

[20] https://scikit-learn.org/stable/modules/generated/sklearn.cluster. KMeans.html (last accessed 05 Jan 2021)

[21] W. M. Spears, V. Anand, "A study of crossover operators in genetic programming," in Methodologies for Intelligent Systems. ISMIS 1991. Lecture Notes in Computer Science (Lecture Notes in Artificial Intelligence), Z. W. Ras, M. Zemankova, Eds. Berlin: Springer, vol 542, pp. 409-418, 1991.

[22] M. Milicevic, K. Zubrinic, I. Obradovic, T. Sjekavica, "Data Augmentation and Transfer Learning for Limited Dataset Ship Classification," WSEAS Transactions on Systems and Control, vol 13, pp. $460-465,2018$.

\section{Creative Commons Attribution License 4.0} (Attribution 4.0 International, CC BY 4.0)

This article is published under the terms of the Creative Commons Attribution License 4.0

https://creativecommons.org/licenses/by/4.0/deed.en_US 